\title{
STUDIES IN LUMINESCENCE.
}

By Edward L. Nichols and Ernest Merritt.

VII. Further Experiments on the Decay of Phosphorescence in Sidot Blende and Certain Other Substances.

I $\mathrm{N}$ the two preceding papers of this series ${ }^{1}$ experiments were described in which the phosphorescence of Sidot blende was followed by the spectrophotometer for about ten seconds after the cessation of excitation. At the end of this period the intensity of phosphorescence had fallen to about four per cent. of its original value. Although it was possible to see the phosphorescent light in the spectrophotometer for a much longer period than this, the extreme faintness of the field made accurate measurements out of the question. In attempting to determine the law of decay throughout a wider range we have therefore been compelled to abandon the use of the spectrophotometer and to measure the total light.

Measurements of the total intensity do not, in general, afford a satisfactory means of determining the law of decay of phosphorescence, since the phosphorescence spectrum usually contains two or more bands, which decay at different rates. We have already referred to the difficulties that are met with in interpreting the results of such measurements. In the case of Sidot blende the conditions are, however, relatively simple, for the phosphorescence of the green band is so much more prominent, both in duration and in intensity, than that of the violet bands that the presence of the latter does not appreciably affect the total intensity. Measurements of the total phosphorescence of Sidot blende are therefore practically measurements of the intensity of the green band only. If all parts of this band decay at the same rate, measurements of total intensity will give the same results as measurements taken at some particular portion of the band. Our earlier observations have shown that all

1 Physical. Review, Vol. 21, p. 247, I905; Vol. 22, p. 279, 1906. 
parts of the green band of Sidot blende do decay at the same rate for at least ten seconds. It seems not unlikely, therefore, that this equality of rates will be maintained.

Although the present paper deals chiefly with the phosphorescence of Sidot blende the results obtained in the study of several other substances are also included.

\section{Experimental Method.}

The apparatus used is shown in Fig. 38. The white screen was removed from a Lummer-Brodhun photometer and the Sidot blende screen, $s$, was put in its place, the active surface of the screen being toward the left. The screen was usually excited by a Lummer mercury lamp, $L$, of the "end on" form. In some instances the carbon arc, and in other cases the spectrum of the carbon arc, was used for excitation. The exciting light could be cut off by means of a shutter, $S$, which at the same time made a record on a chronograph. When the mercury lamp was used in excitation a piece of blue glass, $B$, was placed between the lamp and the screen, so that only the violet lines in the spectrum were used.

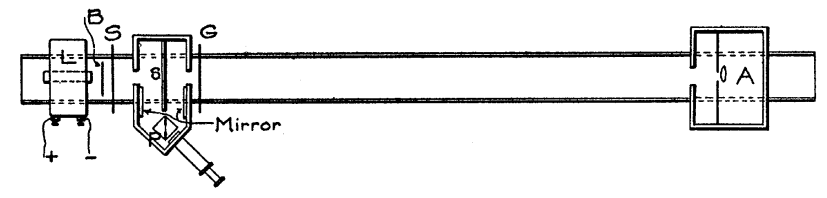

Fig. 38.

The right-hand side of the screen as seen in Fig. 38 was of white paper and could be illuminated by means of the acetylene lamp, $A$. Two pieces of green glass, $G$, served to produce a sufficient color match. The acetylene flame, $A$, was in a metal box, and the light used emerged from a small opening immediately in front of the central part of the flame. Small changes in pressure were therefore without effect upon the intensity of this comparison source.

The procedure in taking observations was as follows :

One observer, with his eyes suitably protected from stray light, watched the decay of the phosphorescence after the shutter had been closed, and recorded by means of a key the instant at which the phosphorescent light and the comparison field were equal. The 
second observer then shifted the position of the comparison flame, $A$, to a slightly greater distance from the photometer, and the time when equality was again reached was recorded as before. In this way it was often possible to get as many as fifteen points on the curve of decay, although a smaller number than this was more usual.

\section{Experiments with Sidot Blende.}

In preliminary experiments the light from the carbon arc was used in excitation. It was thought that simpler conditions would be obtained if exciting light having only a small range of wavelengths was used, and a spectrum of the arc was therefore formed, the violet end of which was used for excitation. The gain in the intensity of phosphorescence which resulted from cutting off the red and infra-red rays was very noticeable.

In our previous experiments with Sidot blende it was found that the decay of phosphorescence during the first seven seconds was in accordance with the law

$$
I=\frac{\mathrm{I}}{(a+b t)^{2}}
$$

so that upon plotting the curves with $T$ and $I^{-\frac{1}{2}}$ as coördinates a straight line was obtained. Since the distance between the photometer screen and the acetylene flame is proportional to $I^{-\frac{1}{2}}$, it was convenient to plot the results of the present experiments in the same way.

The general character of the decay curves when plotted in this manner is exhibited by the curves of Fig. 39. The violet end of the carbon arc spectrum was used in excitation, and the duration of excitation was varied as indicated.

Inspection of Fig. 39 shows that while each curve starts as a line which is nearly straight in the neighborhood of origin, it soon begins to bend over and ultimately changes into a second straight line having a different slant. This behavior is especially noticeable in the case of long exposures.

If phosphorescence is due to the recombination of ions that have been separated by the action of the exciting light, as assumed in the theory of Wiedemann and Schmidt discussed in our last paper, 
it appears that the coefficient of recombination does not remain constant during the whole period of decay. During the first six or seven seconds this coefficient is practically constant, as was shown by the results of our previous experiments. A change then begins which continues for about twenty or thirty seconds, after which the curves indicate a new coefficient, smaller than before. This new coefficient, as is shown by the constant slant of the curves of Fig. 39, remains constant during the rest of the period of decay, or at least until the phosphorescence has become too faint to measure.

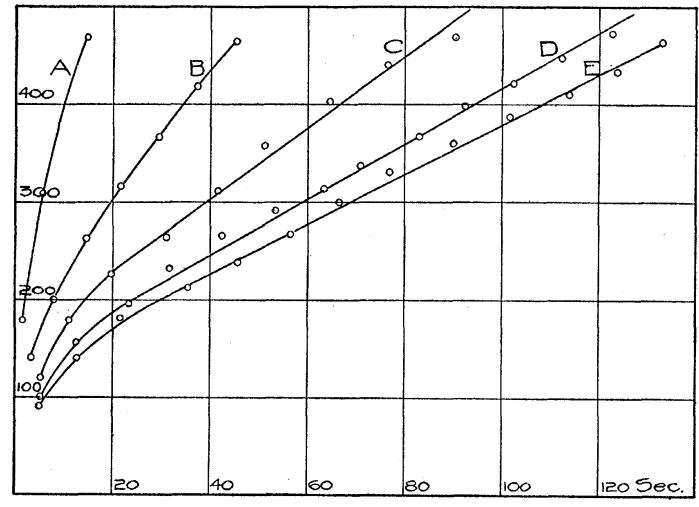

Fig. 39. Effect of duration of excitation. Violet end of carbon-arc spectrum used in excitation. The curves were taken in the order indicated by the letters. The times of excitation were as follows :

$$
\begin{aligned}
& \text { Curve } A, 0.9 \text { sec. } \\
& \text { " } B, 2.4 \mathrm{sec} \text {. } \\
& \text { " } C, 4.5 \mathrm{sec} \text {. }
\end{aligned}
$$

Whatever theoretical interpretation of the curves is adopted it is clear that the decay of phosphorescence involves two distinct processes which merge into one another. In each of these the decay obeys the simple law already referred to, but the rate at which the phosphorescence dies out is greater in the first process than in the second.

It will be observed that the slant of the curve, for each of the two processes, is a function of the duration of excitation.

In Fig. 40 is shown a series of curves taken under the same conditions as those of Fig. 39, except that the mercury arc was used 
in excitation instead of the violet end of the ordinary arc spectrum. One is immediately struck by the difference between the two sets of curves. In Fig. 40 there is a strong tendency toward parallelism in the parts of curves corresponding to the second process. In Fig. 39 no such tendency is observable.

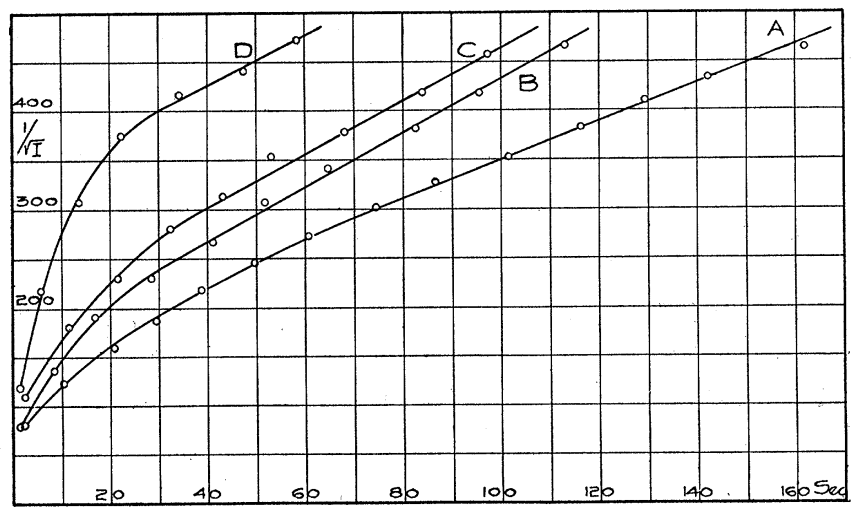

Fig. 40. Effect of duration of excitation. Violet light of mercury arc used for excitation. The curves were taken in the order indicated by the letters. The times of excitation were as follows:

$$
\begin{array}{cccc}
\text { Curve } A, 27 \text { sec. } & \text { Curve } C, 3.1 \text { sec. } \\
\text { " } B, 10 \text { sec. } & \text { “ } D, 1.2 \text { sec. }
\end{array}
$$

We were at first inclined to ascribe this difference to the fact that different kinds of exciting rays had been used in the two cases. The two sets of curves differ however in another respect, namely, in the order in which the curves were taken. In the first case the curves of short excitation were taken first, while in the latter case the curves corresponding to long excitations were the first observed. It appeared possible that the difference in the form of the curves was due to this difference in sequence rather to the difference in exciting light.

To test this matter the observations plotted in Fig. 4I were made. It will be noticed that the dotted curves are similar to those in Fig. 40 while the full curves are similar to those of Fig. 39. The mercury arc was used in excitation in all cases. But curves taken after the screen had been subjected to the long excitations corresponding to Curves $C$ and $D$ differ widely from the curves taken with approxi- 
mately the same excitation previous to $C$ and $D$. A comparison of Curves $A$ and $E$ illustrates this point, the duration of exposure being exactly the same in each of these two cases.

These results indicate that some change is produced in the phosphorescent material by the action of the exciting light, and that this changed condition persists for a considerable period after all visible phosphorescence has ceased. In other words the effect of a given excitation in producing phosphorescence depends upon the previous history of the phosphorescent substance.

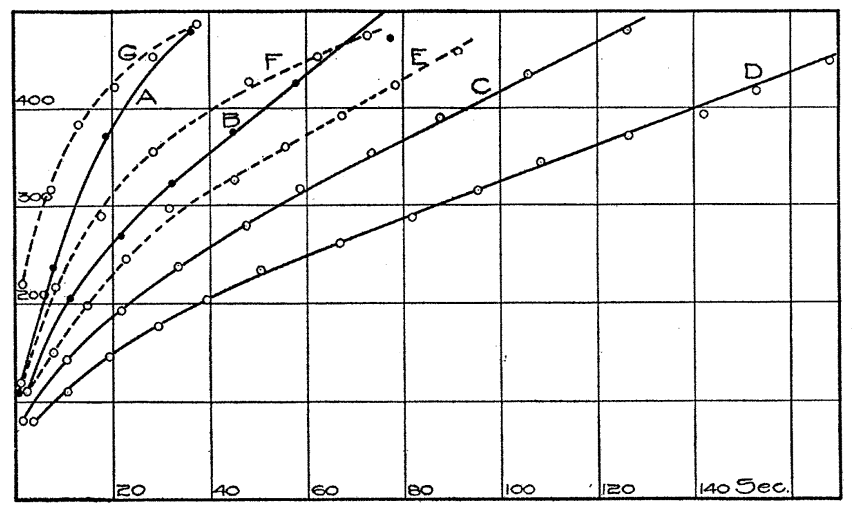

Fig. 41. Effect of duration of excitation. Mercury arc. The curves were taken in the order indicated by the letters. The times of excitation were as follows :

$$
\begin{array}{cccc}
\text { Curve } & A, & 4.3 \text { sec. } & \text { Curve } E, 4.3 \text { sec. } \\
\text { “ } & B, 8.2 \text { sec. } & \text { " } & F, 1.4 \text { sec. } \\
\text { " } & C, 16.0 \text { sec. } & \text { " } & G, 1.0 \text { sec. } \\
\text { " } & D, 56.0 \text { sec. } & &
\end{array}
$$

If the screen is allowed to rest in the dark for a number of hours this semi-permanent effect of exposure in part dies out. But rest alone dose not restore the screen completely, even if continued for several days. The effect of rest was also found to be somewhat uncertain, being much greater on some occasions than on others.

Several methods of restoring the screen to a standard condition were tried. Heating the screen to the temperature of boiling water for several minutes and then cooling it again to the temperature of the room seemed effective. But this method required considerable time and has not been thoroughly tested. Cooling the screen to 
the temperature of liquid air and afterwards warming it gradually to the original temperature seemed to be without effect.

The well known effect of the red and infra-red rays in suppressing the long time phosphorescence of various substances led us to think that these rays might also prove effective as a means of restoring the screen to a standard condition. This conjecture proved to be correct, and exposure to the red and infra-red rays of a $50 \mathrm{c}$. p. lamp at a distance of about $20 \mathrm{~cm}$. was found to be both convenient and satisfactory. A piece of ruby glass placed in front of the lamp served to remove the more refrangible rays. An exposure of a few seconds to the rays that passed through the ruby glass was sufficient to bring the screen into what seemed to be a definite standard condition. A longer exposure was, however, ordinarily used.

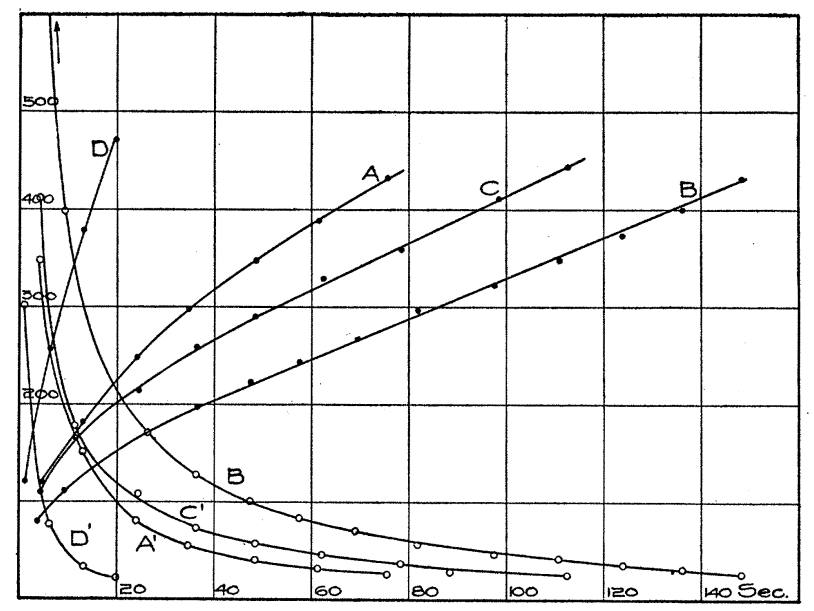

Fig. 42. Illustrating the relative effect of rest and of exposure to infra-red.

$A$, Io sec, excitation after rest of 24 hours in the dark.

$B, 2$ min. excitation.

$C$, ro sec. excitation immediately after $B$.

$D$, ro sec. excitation after exposure of $4 \mathrm{~min}$. to infra-red from 50 candle power lamp.

Curves $A^{\prime}, B^{\prime}, C^{\prime}, D^{\prime}$ correspond to $A, B, C, D$ except that $I$ is plotted in place $I^{-\frac{1}{2}}$.

The change produced by excitation and the effect of the red and infra-red rays are illustrated by the curves shown in Fig. 42. Curve $A$ shows the behavior of the screen when exposed for ten 
seconds after resting in the dark for twenty-four hours. Curve $B$ is that corresponding to an exposure of two minutes; Curve $C$ was taken with an exposure of ten seconds immediately after Curve $B$; and Curve $D$, also with an exposure of ten seconds, was taken after the screen had been exposed to the red and infra-red rays for four minutes. ${ }^{11}$

In some respects the behavior of the screen is analogous to the magnetic behavior of iron. When iron is magnetized a certain residual magnetization remains after the removal of the magnetizing force, and the effect produced by a subsequent magnetizing force depends upon the magnetic history of the specimen. Similarly some change is produced in Sidot blende by excitation which does not immediately disappear upon the removal of the exciting light, and which modifies the effect produced by subsequent excitation.

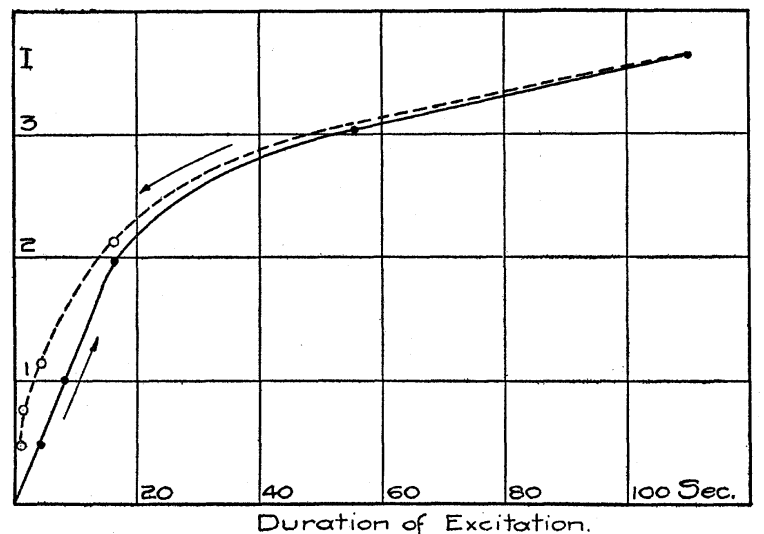

Fig. 43. Hysteresis loop. Ordinates give the intensity of phosphorescence $30 \mathrm{sec}$. after the end of excitation. The curves from which these points were determined were observed in the order indicated by the arrows.

The analogy is rendered more striking if this property of Sidot blende is exhibited in a different manner, as in Fig. 43. In the curve plotted in this figure the abscissa of each point shows the duration of excitation, while the ordinate gives the corresponding intensity of phosphorescence after thirty seconds decay. The resemblance of the curve to a hysteresis loop for iron is striking.

Experiments dealing with the effect of the infra-red rays during and after excitation will form the subject of a separate paper. 
It seems possible that the action of these rays in destroying the residual effect in the phosphorescent substance is similiar to the effect of jarring or tapping in destroying the residual magnetism of a bar of iron. Ignorance of the existence of hysteresis would evidently lead to confusing results in the case of either of these two classes of phenomena. Our delay in recognizing the effect of previous history has in fact made it necessary for us to discard all of our earlier observations.

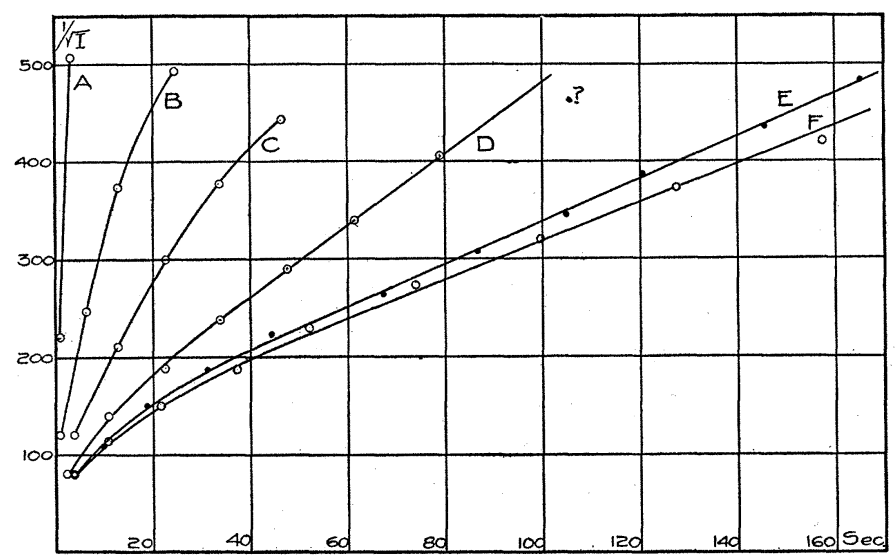

Fig. 44. Effect of duration of excitation. Violet of mercury arc used for excitation. Screen exposed to infra-red for I min. before each curve. The times of excitation were as follows :

$$
\begin{array}{ccccc}
\text { Curve } & A, & 1.2 \text { sec. } & \text { Curve } & D, 37 \text { sec. } \\
\text { " } & B, 5.4 \text { sec. } & \text { " } & E, 60 \text { sec. } \\
\text { " } & C, 12.0 \text { sec. } & \text { " } & F, 15 \text { min. }
\end{array}
$$

In our later work the screen was exposed to the red and infra-red rays as described for one minute before each exposure. With this precaution to avoid the effects of hysteresis the curves shown in Fig. 44 were taken to determine the effect of varying times of exposure.

Since our previous experiments have shown that the curves are accurately straight in the neighborhood of $t=0$, it is possible to determine the initial intensity of phosphorescence by prolonging the curves in each case until they strike the vertical axis. From the intercept thus determined, which is equal to $I_{0}^{-\frac{1}{2}}$, the initial intensity 
can be computed. From the results obtained in this way from the data shown in Fig. 44, Curve $A$ in Figs. 45 and 46 has been plotted. In these two figures $I$ has been plotted instead of $I^{-\frac{1}{2}}$.

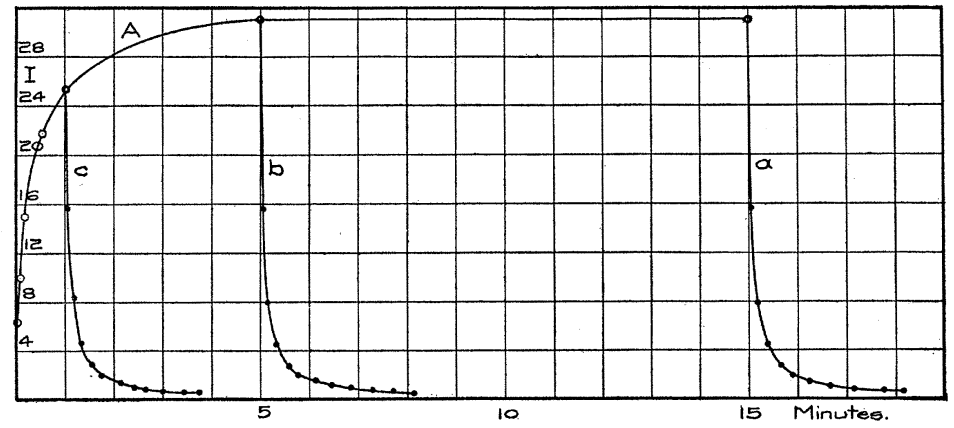

Fig. 45. Effect of duration of excitation. Curve $A$ shows the initial intensity of phosphorescence $(I)$ as a function of the time of excitation. Curves $a, b$ and $c$ show the decay of phosphorescence after excitations of $15 \mathrm{~min}$., $5 \mathrm{~min}$. and $\mathrm{I}$ min., respectively.

It will be noticed that the intensity of phosphorescence at first increases rapidly with increased duration of exposure, but that after an exposure of two or three minutes is reached there is little further change. The phosphorescence may be said to be saturated so far as the effect of duration of excitation is concerned. 'Not only is the initial intensity unaltered by longer excitation, but the form of the decay curve also remains constant, as is indicated by Curves $a$ and $b$ in Fig. 45. (These curves correspond to Curves $F$ and $E$ of Fig. 43.)

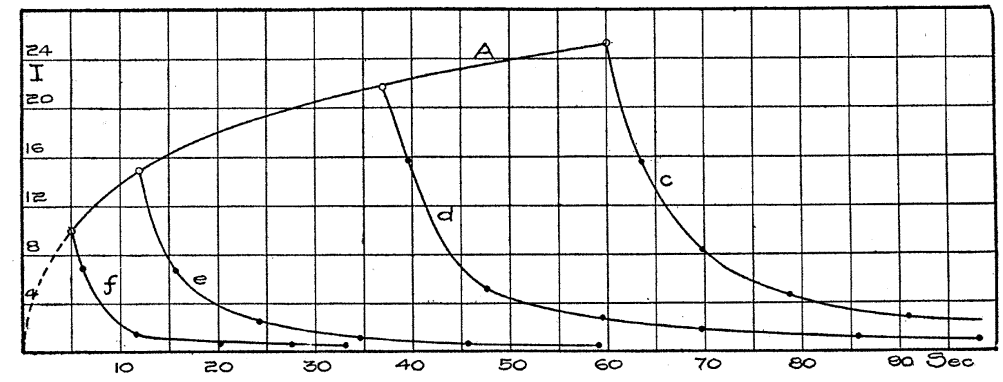

Fig. 46. Effect of duration of excitation. A portion of the same curve shown in Fig. 45 plotted to a larger scale.

We have also studied to some extent the influence of the intensity of the exciting light upon the form of the decay curves. In order 
to vary the intensity of the exciting light several metal stops were prepared, which could be placed immediately in front of the mercury lamp. The apertures of these varied from one millimeter in diameter to the full size of the mercury lamp tube, namely, I $5 \mathrm{~mm}$. To determine the intensity of the exciting light corresponding to each of these the following photometric method was used: The phosphorescent screen was removed from the photometer and a piece of white cardboard was put in its place. This being illuminated by the violet light from the mercury arc passing through the stop whose constant was to be determined, the intensity was measured by shifting the position of the comparison flame on the opposite side of the photometer. Suitable glass screens were used to equalize the colors on the two sides. To avoid errors resulting from the flickering of the mercury arc ten settings were made for each determination.

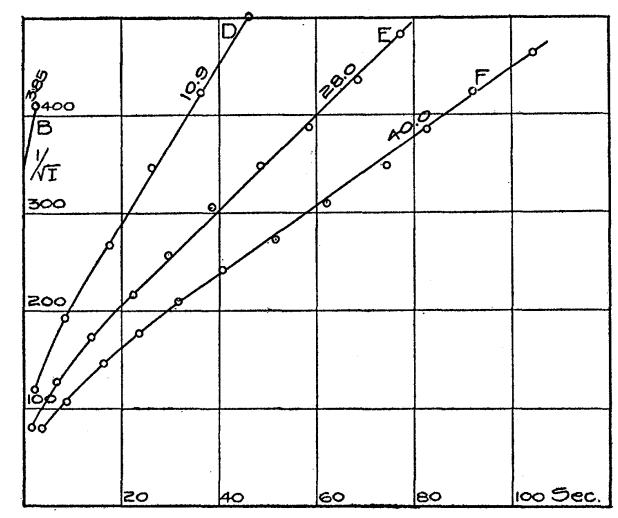

Fig. 47. Effect of varying the intensity of the exciting light. Exposure in each case $20 \mathrm{sec}$. The relative intensity of the exciting light is marked on each curve.

In Fig. 47 decay curves are shown for different intensities of the exciting light, the excitation in each case lasting for twenty seconds. In Figs. 48 and 49 similar sets of curves are shown for which the excitations were respectively 40 seconds and 2 minutes.

A study of these curves shows that there is some approach to intensity saturation; in other words, the intensity of phosphorescence is nearly proportional to the intensity of the exciting light for small values of the latter, but increases less rapidly than the exci- 
tation when the exciting light is strong. This point is well brought out by Curve $A$ in Fig. 50, in which the ordinates are proportional to the initial intensity of phosphorescence, $I_{0}$. The values of $I_{0}$ were determined from the data of Figs. 47, 48 and 49 by extrapola-

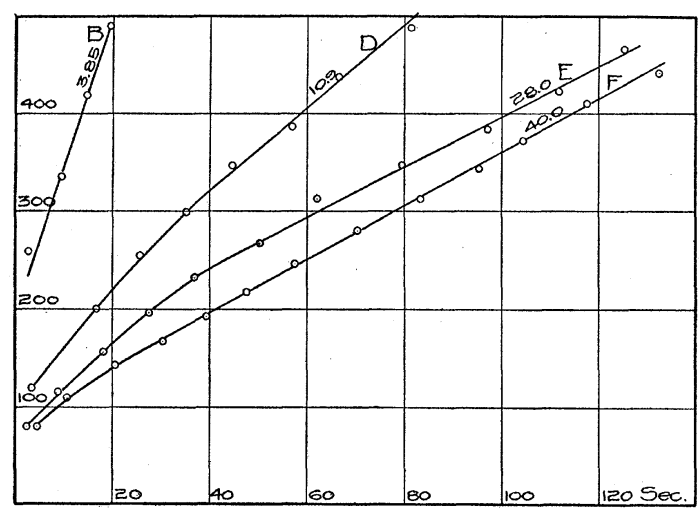

Fig. 48. Effect of varying the intensity of the exciting light (as indicated on each curve). Exposure in each case 40 sec.

tion, upon the assumption that the relation between $t$ and $I^{-\frac{1}{2}}$ is linear for small values of $t$. Since the early portion of each decay curve is chiefly determined by the first two or three points, which are the most difficult to observe, the values plotted for $I_{0}$ are subject to considerable error. Curve $A$ is nevertheless reasonably

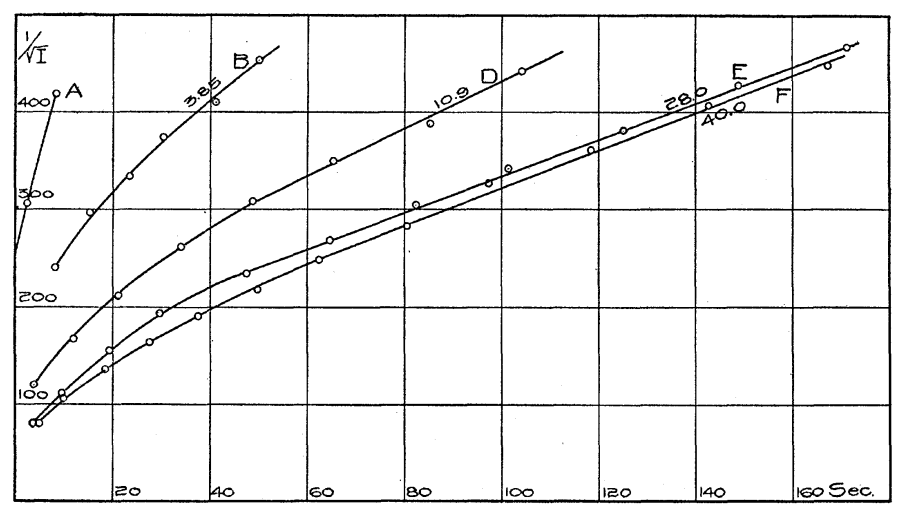

Fig. 49. Effect of varying the intensity of the exciting light (as indicated on each curve). Exposure 2 min. in each case. 
smooth and indicates nearly exact proportionality between intensity of excitation and initial phosphorescence. It is only with the most intense excitation used that saturation begins.

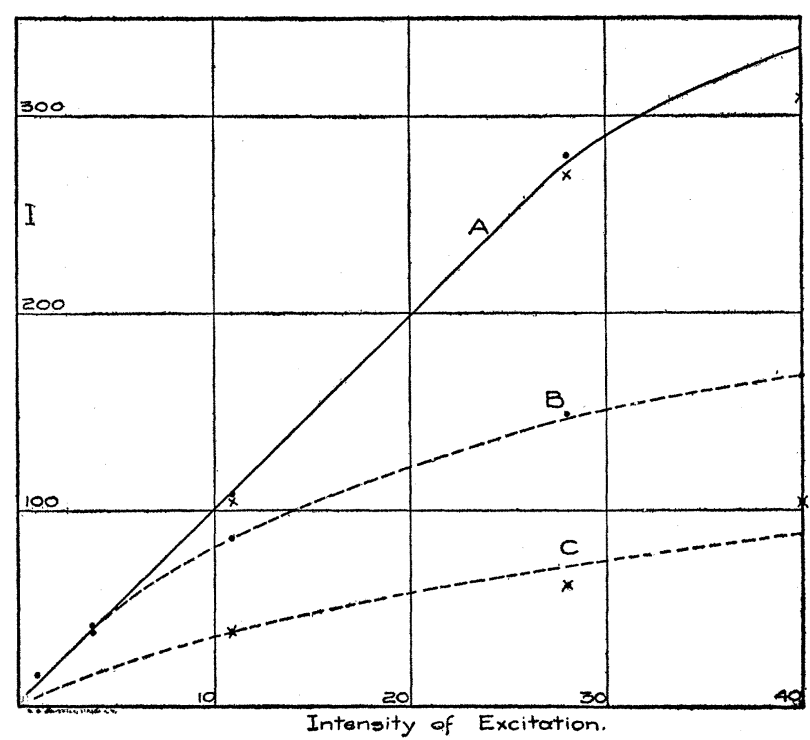

Fig. 50. Curve $A$. Effect of the intensity of the excitation upon the initial intensity of phosphorescence. The points marked by dots are for an exposure of $2 \mathrm{~min}$.; those indicated by crosses are for an exposure of $20 \mathrm{sec}$. In Curves $B$ and $C$ the ordinates show the intensity of phosphorescence $\mathrm{I}$ min. after excitation had ceased.

It is to be observed that in most cases the values of $I_{0}$ that are computed from the data of Fig. 47 lie on the same curve as those obtained from the data of Fig. 49. A well-marked difference is noticeable only in the case of the points corresponding to intense excitation. In other words, for weak excitations the intensity of the initial phosphorescence is the same after an exposure of 20 seconds as for one of two minutes. There at first appears to be a contradiction here to the results shown in Figs. 45 and 46 . But while this may be due to the uncertainty in the values of $I_{0}$, it is readily explained upon the assumption that a weak excitation produces its full effect more promptly. The form of the curves shown in Figs. 45 and 46 is probably largely dependent upon the intensity of the exciting light. 
The ordinates of Curves $B$ and $C$ (Fig. 50) show the intensity of phosphorescence one minute after the exciting light was cut off. For Curve $B$ the exposure was two minutes, while for Curve $C$ the exposure was twenty seconds. The effect of duration of exposure is here well marked.

In the case of exposures lasting for several seconds or more the phenomena are manifestly complicated by the fact that the semipermanent change, to which we have already referred, is taking place in the active substance during the time of excitation. It seemed probable that the relation between intensity of excitation and intensity of phosphorescence might prove simpler if the duration of excitation was reduced to a minimum. A series of curves was therefore taken with a spark as exciting source. Preliminary trials showed that the most intense excitation was furnished by discharging eight large jars through a spark gap about $2 \mathrm{~cm}$. long with one cadmium terminal. The distance of the spark gap from the screen was varied from about $10 \mathrm{~cm}$. to $35 \mathrm{~cm}$. A single spark at IO $\mathrm{cm}$. distance gave an excitation approximately equivalent to thirty seconds exposure to the mercury arc.

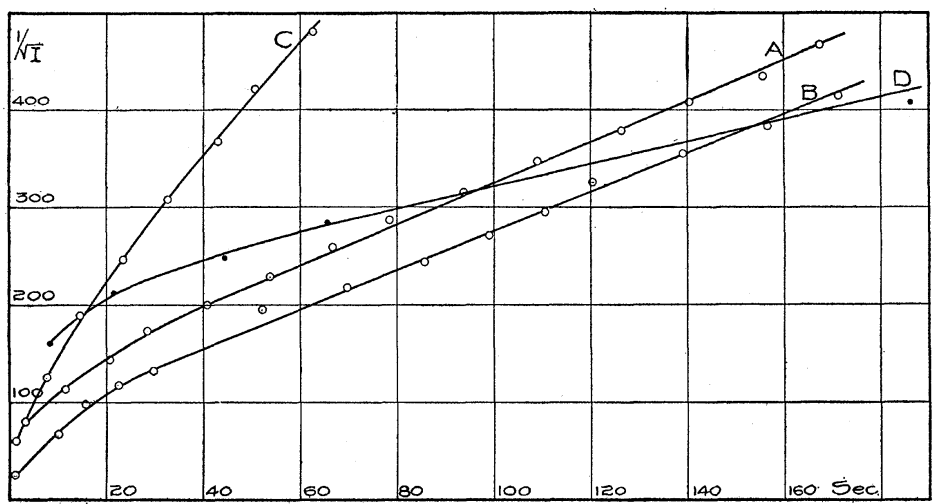

Fig. 51. Typical decay curves for various substances. Curve $A$, Sidot blende ; Curve $B$, "Emanations-pulver" ; Curve $C$, Willemite; Curve $D$, Balmain's paint.

Experiments with the practically instantaneous excitation produced by a single spark showed that the phosphorescence was proportional to the intensity of excitation, not merely initially but 
throughout the whole period of decay. In other words, it was possible to bring a decay curve determined with the spark at a distance $d_{2}$ into coincidence with the curve corresponding to the distance $d_{1}$ by multiplying each ordinate $I_{2}$ by the ratio $d_{2}^{2} / d_{1}^{2}$.

\section{Experiments with Different Phosphorescent Substances.}

It is natural to inquire whether the complex phenomena exhibited by Sidot blende are peculiar to this particular material, or whether its behavior is typical for a large class of phosphorescent substances. In order to test this matter we have determined the decay curves under similar conditions with three other substances, namely, "Emanations-pulver," " willemite and Balmain's paint. Characteristic curves for these three substances together with a representative curve for Sidot blende are shown together in Fig. $5 \mathrm{I}$. It will be noticed that the curves are all of the same type. In each case the decay is at first rapid and apparently according to the same law that was found to hold during the early stages of decay in the case of

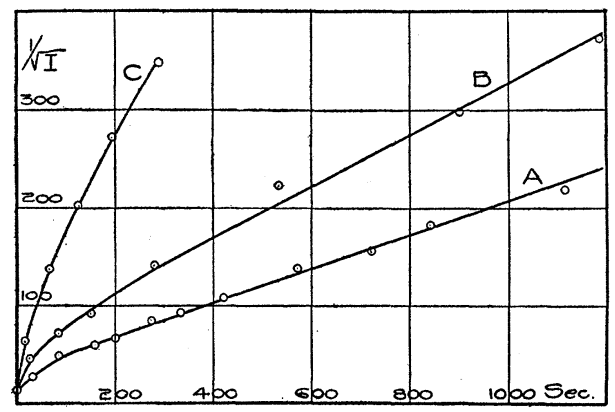

Fig. 52. Decay curves plotted from the data of E. Becquerel. (Ann. de Chimie et de Physique, series 3, Vol. 62, 1861.)

Curve $A$, Sulfure de calcium lumineux vert (2, p. 69).

“ $B$, Sulfure de strontium lumineux vert (p. $7 \mathrm{r}$ ).

“ $C$, Sulfure jaune-orangé vif (p. 68).

Sidot blende. After twenty or thirty seconds the curves begin to bend, and finally become straight lines whose slant is less than that of the earlier part of the curve.

1 Obtained from Leppin and Masche, who do not state the composition of the powder. It is said to be especially sensitive to the influence of the radioactive emanations. 
Upon plotting the data of $\mathrm{E}$. Becquerel ${ }^{1}$ in the same manner we find that in several cases the curves are of exactly the same type as those obtained by us. Three such curves are shown in Fig. 52. In fact all the data recorded by Becquerel in his classic papers on this subject give curves which show the samie general characteristics, although in several instances the curves are not so smooth as those shown in this figure.

It is interesting to note also that the data of Darwin ${ }^{2}$ on Balmain's paint give a curve similar to those obtained by us with this substance when plotted in the same way. Two decay curves taken by E.

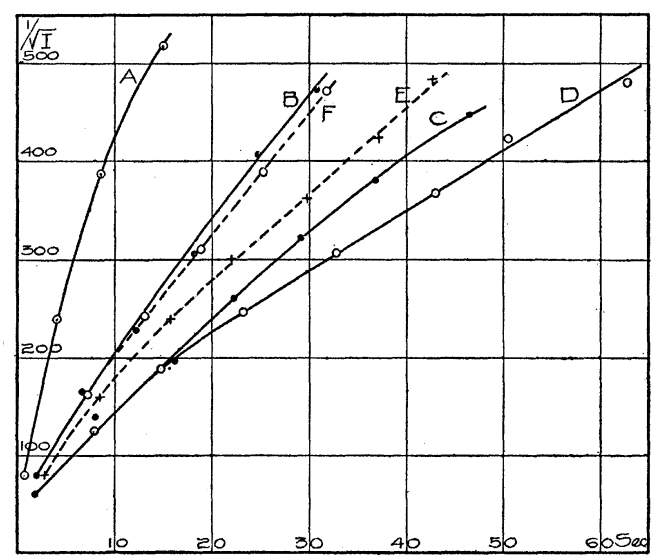

Fig. 53. Willemite. Excited by a spark between iron terminals. The curves were taken in the order indicated by the letters, and with the excitations stated below :
Curve $A, 0.7 \mathrm{sec}$.
“ $B, 3.4 \mathrm{sec}$.
“ $\quad B, 3.4 \mathrm{sec}$.
Curve $D, 60.0 \mathrm{sec}$.
“ $E, 3.9 \mathrm{sec}$.
" $F, 1.0 \mathrm{sec}$.

Wiedemann ${ }^{3}$ with Balmain's paint also show the same characteristics.

It appears therefore that the decay curve for Sidot blende is similar in its main features to the curves obtained for a large number of other phosphorescent substances. In fact we do not know of

1 Becquerel, La Lumière. Also Annales de Chimie et de Physique, Series 3, Vol. 62, 1861 .

'2 Darwin, Philosophical Magazine, Vol. II, p. 209, I88I.

" ${ }^{\mathrm{E}}$. Wiedemann, Zur Mechanik der Leuchtens, Wied. Ann., Vol. 37, p. 177 I889. 
any case of long time phosphorescence which shows a different type of curve.

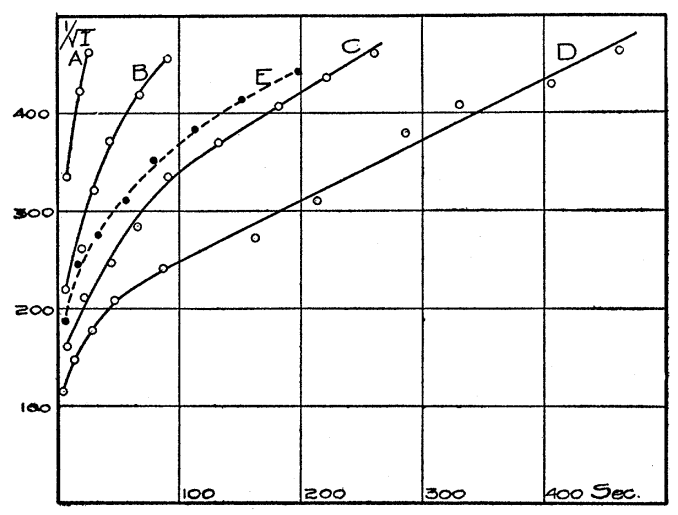

Fig. 54. Balmain's paint. The curves were taken in the order indicated by the letters, and with the following excitations :

$$
\begin{array}{ccc}
\text { Curve } A, & 5.9 \text { sec. } & \text { Curve } D, 42.0 \text { sec. } \\
\text { " } B, 12.0 \text { sec. } & \text { " } E, 6.4 \text { sec. } \\
\text { " } C, 26.0 \text { sec. } & &
\end{array}
$$

The peculiar behavior of Sidot blende which we have compared with magnetic hysteresis is also exhibited by willemite and Balmain's paint, as illustrated in Figs. 53 and 54 . In each of these cases it is evident that the effect of a given excitation is dependent on the previous history of the substance. We have not yet had an opportunity to test this phenomenon in other substances.

\section{Summary.}

The most important points brought out by the experiments here described may be briefly stated as follows :

I. Form of Decay Curve. - The curve obtained by plotting the values of $I^{-\frac{1}{2}}$ as ordinates and the corresponding values of $t$ as abscissas is a straight line for small values of $t$; it changes to a curve concave toward the axis of $t$ as $t$ increases; but for still larger values of $t$ the relation between $I^{-\frac{1}{2}}$ and $t$ is again linear, and remains so until $I$ becomes too small to measure. In other words the decay curve, when plotted in this way, consists of two straight portions which gradually merge into one another. 
2. Effect of Duration and Intensity of Excitation. - Not only the intensity of phosphorescence, but also the form of the decay curve, is dependent on the intensity and duration of excitation. The slant is altered in each of the straight parts of the curve by changing either of these two factors in the excitation.

3. Hysteresis. - The behavior of the phosphorescent substance with a given excitation depends upon its previous history. Some semi-permanent change is produced by excitation which persists for several hours, or even for several days, after visible phosphorescence has ceased.

4. Effect of Red and Infra-red Rays. - In the case of Sidot blend the semi-permanent condition produced by excitation may be destroyed and the screen restored to a standard state by brief exposure to the red and infra-red rays. We have not yet determined whether the same effect is produced by the long waves in the case of other phosphorescent substances. 